\title{
La expansión de la microempresa informal: ¿cuestión de desempeño o de empeño?
}

\author{
The expansion of informal microenterprises: \\ Is it a matter of performance or determination? \\ José G. Aguilar Barcelón ${ }^{1}$ Pedro P. Orraca Romano ${ }^{2}$ \\ ${ }^{1}$ Universidad Autónoma de Baja California, México \\ ${ }^{2}$ El Colegio de la Frontera Norte, México
}

Recibido el 3 de noviembre de 2019; aceptado el 6 de agosto de 2020

Disponible en Internet el: 2 de septiembre de 2020

\section{Resumen}

Los resultados acerca de la relación entre la gestión del emprendimiento informal y sus posibilidades de expansión son aún escasos. Con base en datos de la ENIGH y a través de regresiones logísticas, se exploran las características asociadas al crecimiento de este tipo de emprendimientos en México. Se comprueba que el establecer el primer local o evolucionar hacia el segundo son problemas estructuralmente distintos y no siempre asociados a indicadores económico-financieros. Aunque fundamentales para cumplir con sus objetivos, las utilidades no parecen ser el principal detonador del crecimiento en este sector. Lo anterior podría deberse a la falta de orientación a la maximización intertemporal de beneficios y a las restricciones que impone un proceso de crecimiento en el nivel de utilidades de corto plazo. Por lo tanto, además de los indicadores económico-financieros, otras características asociadas al emprendedor y la naturaleza de su proyecto deben ser tomadas en cuenta para entender el potencial de crecimiento de este.

\footnotetext{
*Autor para correspondencia

Correo electrónico: gaba@ uabc.edu.mx (J.G. Aguilar Barceló).

La revisión por pares es responsabilidad de la Universidad Nacional Autónoma de México. 
Código JEL: D21, E26, L26

Palabras clave: Microempresa; Informalidad; Desempeño; Crecimiento; Expansión

\begin{abstract}
The results on the relationship between the management of informal entrepreneurship and their possibilities of expansion are still scarce. Based on ENIGH data and through logistic regressions, we explore the characteristics associated with the growth of these enterprises in Mexico. It is shown that establishing the first location or evolving into a second one are structurally different problems, which are not always linked to economic-financial indicators. Although vital to meet their objectives, profits do not seem to be the main trigger for growth in this sector. This could be due to the lack of orientation towards intertemporal profit maximization and the restriction imposed by a growth process on the level of short-term profits. Therefore, in addition to conventional economic-financial indicators other characteristics associated with the entrepreneur and the nature of his project must be considered to understand its growth potential.
\end{abstract}

JEL Code: D21, E26, L26

Keywords: Microenterprise; Informality; Performance; Growth; Expansion

\title{
Introducción
}

Entre las razones por las que los gobiernos fomentan la creación y consolidación de microempresas 1 a través de programas de financiamiento y asistencia técnica está su potencial efecto positivo en la estabilización de la economía (Texis, Ramírez y Aguilar, 2016). Lo anterior es particularmente reconocido en periodos de recesión económica, cuando las microempresas juegan un papel central como mecanismo contracíclico de reactivación del mercado interno (Mungaray, Ramírez, Aguilar y Beltrán, 2007). No obstante, la vulnerabilidad y escaso crecimiento de estos proyectos a lo largo de su vida, incluso siendo rentables, hace que en ocasiones se cuestione su contribución al desarrollo local (Docquier, Müller y Naval, 2014).

Aunque se ha investigado profusamente acerca de los factores que impulsan el desempeño de las microempresas en México (Mungaray et al., 2007; Hayes, Chawla, y Kathawala, 2015; Dini y Stumpo, 2018; Rivera, 2018), los resultados acerca de la relación entre este y el potencial de expansión son aún escasos (Allinson, Braidford, Houston y Stone, 2013; Jang y Park, 2011; Federico y Capelleras, 2015; Fuertes-Callén y Cuellar-Fernández, 2019); no se

${ }^{1}$ Llamadas también "emprendimientos" o "negocios de hogar" en este documento. 
diga ya si la búsqueda se circunscribe al ámbito del emprendimiento informal, que en México no es un tema menor. Según datos del Instituto Nacional de Estadística y Geografía (INEGI), los propietarios de emprendimientos que operan en la informalidad ya sean empleadores o trabajadores por cuenta propia, representan el $23.1 \%$ de la población económicamente activa (INEGI, 2018).

Que la expansión solo se dé en un reducido porcentaje de los proyectos microempresariales podría tener múltiples causas coexistentes, tales como las limitaciones de la demanda, la falta de acceso al financiamiento o la presión por el uso de los excedentes, pero también otras atribuibles a la visión del promotor, como lo son el cumplimiento de expectativas (al haberse alcanzado metas más inmediatas), la falta de implementación de controles o la aversión a los compromisos a largo plazo.

Así, dada la importante presencia, pero también, el complejo panorama del sector microempresarial en México -incluido el de tipo formal- resulta imperante identificar las circunstancias bajo las cuales el camino de la expansión es eventualmente explorado, al margen del entorno macroeconómico. No se puede obviar que esta expansión puede llegar constituir una vía de desarrollo económico comunitario que vaya más allá de los efectos directos en la calidad de vida de los propietarios y sus familias, contraviniendo así, el enfoque tradicional por el que el emprendimiento informal solo es una salida urgente y fugaz a las limitaciones de liquidez.

La presente investigación se propone, por una parte, identificar y caracterizar los emprendimientos de baja escala productividad de acuerdo con su tamaño y las visiones del futuro de sus propietarios, y por la otra, medir las consecuencias de algunas acciones de gestión y de resultados económico-financieros en las decisiones de expansión de estos emprendimientos tomando en consideración su naturaleza y nivel de desarrollo. El trabajo explora el crecimiento desde un enfoque operativo y motivacional, basado en la realidad mexicana y de muchas economías en vías de desarrollo, y no desde uno estructurado y sistémico (que, por ejemplo, depende de la formalización). Así, la hipótesis que se plantea es que la posibilidad de crecimiento de la microempresa informal (y con ello, su potencial formalización y evolución en la estratificación empresarial), aunque pueda estar relacionada con sus resultados económico-financieros, también estaría vinculada de forma significativa a sus condiciones iniciales, estructura y visión del promotor.

El estudio se organiza en cinco secciones incluyendo esta introducción. En la siguiente se presenta el marco teórico, destacando las principales dificultades de la microempresa para crecer y expandirse, así como la importancia de identificar la naturaleza y circunstancias de los emprendimientos para poder anticipar su evolución. Posteriormente se describen tanto 
la metodología como la fuente de información empleada. Después, en el apartado de resultados, se examina el comportamiento estadístico de las variables y se analizan y discuten las estimaciones econométricas a la luz de la literatura. Por último, se desarrollan las principales conclusiones y limitaciones del estudio.

\section{Marco teórico}

Los factores del entorno sin duda inciden en los indicadores de resultados de una microempresa. De acuerdo con el INEGI (2016a), el 39.7\% de los microempresarios asegura que las características de la demanda, la marginación económica, el exceso de competidores o una agresiva competencia en precios restringen sus márgenes, haciendo imposible incrementar el volumen de ventas sin incurrir, al menos temporalmente, en pérdidas que generalmente no están dispuestos a soportar.

Es común también que el sector microempresarial regulado atribuya la imposibilidad de crecimiento a la informalidad en la que operan muchos de los competidores, lo que les estaría permitiendo ofrecer precios más bajos por ahorros derivados de reducciones en la calidad y la evasión de impuestos. Al respecto, Mungaray et al. (2007) encontraron que, en la medida en que las actividades informales sean una práctica socialmente legitimada, aumenta la posibilidad de que también sean lucrativas, al haber menos riesgo de "castigos" por parte del mercado de consumo, o de sanciones por parte de las autoridades. No obstante, el hecho de que este sector alcance una masa crítica suficiente también puede provocar que los intentos de expansión ocurran solo excepcionalmente (Berrone, Gertel, Giuliodori, Bernard y Meiners, 2014; Webb, Tihanyi, Ireland y Sirmon, 2009).

En materia de gestión, entendida como el conjunto de acciones que se llevan a cabo de forma consistente para dirigir y administrar un emprendimiento, suele haber diferencias según el uso de los factores productivos, las barreras de entrada y salida, y por el sector económico de pertenencia (Cruz, López, Cruz y Meneses, 2016). Por ejemplo, mientras que 20.9\% de las empresas de servicios invierte en capacitación, esto solo lo hace el $13.3 \%$ de las manufactureras, y el 7.3\% de las dedicadas al comercio. A su vez, las razones para no invertir en capacitación varían según el estrato empresarial. Las microempresas suelen argüir que sus conocimientos son adecuados para el desarrollo de la actividad, mientras que el costo de interrumpir la producción es el inhibidor habitual en las empresas de mayor tamaño (INEGI, 2016b).

$\mathrm{El}$ análisis particular de los negocios de baja escala productiva muestra que en muchas ocasiones estos llegan a tener una gestión de tipo autocrático donde además el propietario es quien lleva a cabo muchas de las tareas tanto administrativas como operativas. En con- 
secuencia, la evolución de estos emprendimientos es altamente dependiente del discurrir de la vida del propietario (en el sentido de que las decisiones en ambas esferas de acción están asociadas) así como de su experiencia, competencia y ambición (Allinson et al., 2013; Schindler, 2016). Dado que el tiempo dedicado a la actividad microempresarial compromete el de otras actividades individuales y familiares, una búsqueda miope del uso óptimo de los recursos difícilmente asignará suficiente tiempo a la planeación, lo que representa un fuerte obstáculo al crecimiento ${ }^{2}$.

Sumado a lo anterior, Berrone et al. (2014) afirman que el tiempo de dedicación a la microempresa y la continuidad en su operación son determinantes en su desempeño, al grado de que, en el caso de estos emprendimientos, tener que apartarse temporalmente de la ejecución de la actividad pone en riesgo la continuidad del proyecto en su conjunto. En esta lógica, los negocios de hogar tienden a operar según como sea entendido el manejo de los procesos por parte del propietario (Kuratko, Hornsby y Naffziger, 1999). Lo anterior hace evidente que la búsqueda del éxito, por un lado, de la microempresa, y por el otro, de su propietario, pueden ser propósitos con vías de acción divergentes en el corto plazo.

En esta línea, autores como Allinson et al. (2013) exploran la visión de los propietarios acerca del crecimiento de sus emprendimientos y reconocen la existencia de mitos que racionan, quizás injustificadamente, el deseo de crecer. A este respecto, Korunka, Kessler, Frank y Lueger (2011) afirman que apenas la mitad de las iniciativas de autoempleo agregan empleados a la plantilla después de ocho años de operación, mientras que Allinson et al. (2013) encuentran una proporción similar para las pequeñas empresas con empleados tras cinco años de actividad, independientemente del apoyo que hayan recibido durante ese tiempo. Lo anterior sustenta la sospecha de que pasar de un formato de trabajo unipersonal a uno que requiere coordinación entre individuos podría ser altamente desafiante para el propietario y que una "fase de crecimiento" (en términos del número de empleados) no sería de rigurosa observancia en el ciclo de vida de un emprendimiento.

No obstante, cuando se alcanza esta evolución, el microempresario tiende a emplear personal con habilidades generales y no especializadas (Simpson, 2001), llegando a ser incrédulo ante la posibilidad de que otros puedan tener sus competencias. Asimismo, existe la creencia de que capacitar a los empleados es riesgoso debido a que ello supone la adquisición de habilidades que podrían representar competencia directa (Allinson et al., 2013). Finalmente, la sospecha de que contar con más personal representa la entrada a regímenes fiscales de mayor carga impositiva y regulatoria, llega a ser un disuasivo para el crecimiento.

\footnotetext{
${ }^{2}$ Las decisiones cotidianas del individuo suelen derivar de evaluaciones de corto plazo por estar asociadas a necesidades inmediatas.
} 
Berge, Bjorvatn y Tungodden (2015) advierten que las malas decisiones en materia de capital humano pueden constituir un freno para el desarrollo de la microempresa incluso más importante que la falta de financiamiento. En consecuencia, la forma en que el microempresario enfrente estos y otros los retos operacionales (por ejemplo, la planeación estratégica y la implementación de controles operativos), podría determinar si el proyecto se sitúa o no en una potencial ruta de crecimiento (Hayes et al., 2015). A este respecto, autores como Thapa (2015), Markman, Baron y Balkin (2005) y Holm, Opper y Nee (2013) revelan que ciertos factores relacionados con el propio emprendedor, tales como la habilidad de gestión, la necesidad de logro y autonomía, la perseverancia y la tolerancia a la incertidumbre son clave en el desarrollo del emprendimiento.

De cualquier forma, según la Encuesta Nacional de Productividad y Competitividad Empresarial (ENAPROCE), en 2016 el 14.7\% de los microempresarios manifestó que no quería ver crecer su negocio. El 28.4\% atribuía esta negativa a la inseguridad; el 25.1\%, a que se sentía satisfecho con los resultados alcanzados; el 18.6\%, a que anticipaba complicaciones administrativas; y el 16.6\%, a que no quería pagar más impuestos o enfrentar trámites costosos (INEGI, 2016a). Los resultados de esta misma encuesta para 2019 son incluso más inquietantes al mostrar que hasta el $22.5 \%$ de los microempresarios no deseaba ver crecer su negocio. El argumento de $47.4 \%$ de estos era que se sentían satisfechos con los resultados obtenidos hasta ese momento (INEGI, 2019). Lo hasta aquí expuesto permite conjeturar que, más allá de la expectativa de crecimiento soportada en indicadores económico-financieros, en ocasiones puede presentarse una negación originaria por parte del propietario a que este crecimiento ocurra.

Al analizar la direccionalidad de la relación entre crecimiento y buen desempeño económico-financiero en el ámbito de la microempresa, tanto Federico y Capelleras (2015) como Fuertes-Callén y Cuellar-Fernández (2019) sugieren que la primera de las circunstancias repercute positivamente en la segunda, pero no necesariamente al revés. Los autores precisan que la relación depende de los atributos de la empresa y el sector productivo al que esta pertenezca (Federico y Capelleras, 2015), y que incluso un contexto económico adverso no es impedimento para la expansión, siempre que se aborden de forma estratégica las condiciones hostiles (Fuertes-Callén y Cuellar-Fernández, 2019). Monge y Torres (2015) aseguran que las empresas más jóvenes y pequeñas son las que tienden a mostrar mayores incrementos en sus indicadores de desempeño a corto plazo, pero también, más dificultades para evolucionar en la estratificación empresarial. De acuerdo con ellos, de las microempresas que alcanzan 10 años en operación bajo un régimen formal, solo el $5.3 \%$ evoluciona a pequeña, siendo previsible un valor más bajo en un contexto de informalidad. 
De acuerdo con Berrone et al. (2014), bajo una visión funcional de la microempresa, la imperfecta correspondencia entre su desempeño y su potencial de crecimiento podría ser explicada en parte por las motivaciones que le dan origen a esta y que influyen en la forma de gestión utilizada. Estas motivaciones pueden fundamentarse, de acuerdo con Allen, Elam, Langowitz y Dean (2008) y Dawson y Henley (2012), en la necesidad económica urgente (factores de tipo push) o en el aprovechamiento de una oportunidad de mercado (factores de tipo pull). Con énfasis en la informalidad, Berner, Gomez y Knorringa (2008) distinguen entre dos clases de emprendimiento que guardan cierto paralelismo con las anteriores: la de "subsistencia" y la de "orientación a la acumulación".

Autores como Karlan y Zinman (2011), Berge et al. (2015) y Thapa (2015) exponen que la percepción de aquello que garantiza un excedente económico no siempre es compatible con la búsqueda del crecimiento empresarial, y que una conciliación entre los enfoques dependerá de las habilidades de gestión y las relaciones sociales del propietario, pero, sobre todo, de los elementos asociados a su conducta y visión de negocio. En este sentido, existe una inclinación en las empresas de subsistencia a primar la diversificación sobre la especialización y el desarrollo vertical, por lo que, aunque las condiciones del mercado y la madurez conseguida por el paso del tiempo posibiliten la exploración de la alternativa de expansión, la falta de desarrollo de ventajas competitivas dificulta que los microempresarios de subsistencia logren superar la "cultura de la pobreza" (Berner et al., 2008). No obstante, sería arriesgado afirmar que tener objetivos distintos a los de la maximización de beneficios (propios de las microempresas orientadas a la acumulación) anule las posibilidades de crecimiento.

\section{Metodología y datos}

Debido a los objetivos que se persiguen en este trabajo y a la naturaleza transversal de los datos empleados, se recurre al análisis de regresión logística binomial como alternativa para contrastar estadísticamente la hipótesis de investigación. Trabajos como los de Texis et al. (2016),_Berrone et al. (2014) y Monge y Torres (2015) han empleado este modelo para alcanzar objetivos afines a los de la presente investigación. En el primero de estos trabajos se analiza la posibilidad de que las microempresas de base social superen los primeros años de operación; en el segundo se estudian los determinantes del desempeño microempresarial con énfasis en el efecto del capital humano; la última de las referencias explora la dinámica de entrada y salida del mercado de los emprendimientos, así como su crecimiento. 
Para definir el modelo Logit se utiliza la siguiente especificación:

$$
Y=\frac{1}{1+e^{-\alpha-\sum_{i=1}^{k} \beta_{i} X_{i}}}+\varepsilon=\frac{e^{\alpha+\sum_{i=1}^{k} \beta_{i} X_{i}}}{1+e^{\alpha+\sum_{i=1}^{k} \beta_{i} X_{i}}}+\varepsilon
$$

donde $Y$ es la variable dependiente de respuesta binaria $(0,1)$, mientras que $X_{i}(i=1,2, \ldots, k)$ es cada una de las $k$ variables explicativas. Además, $\alpha$ y $\beta$ son parámetros por estimar, siendo este último un vector de tamaño $k$. El modelo Logit presenta una aproximación a la probabilidad de ocurrencia del acontecimiento objeto de estudio $(Y=1)$; en este caso, el incremento en una unidad del número de establecimientos, locales comerciales o puntos de venta (dado que se cuenta con hasta uno de estos), en un periodo determinado. En este trabajo se utiliza el incremento en el número de establecimientos como proxy del crecimiento microempresarial ${ }^{3}$.

Una interpretación más sencilla de los parámetros empleados se obtiene a través de la linealización del modelo. Para ello se define $M$ como la probabilidad de ocurrencia de la opción 1 de la variable dependiente y se expresa como

$$
E(Y)=\operatorname{Pr}(Y=1)=M=\frac{e^{\alpha+\sum_{i=1}^{k} \beta_{i} X_{i}}}{1+e^{\alpha+\sum_{i=1}^{k} \beta_{i} X_{i}}}
$$

que se puede transformar en

$$
\frac{M}{1-M}=e^{\alpha+\sum_{i=1}^{k} \beta_{i} X_{i}}
$$

El cociente entre la probabilidad de ocurrencia del evento sujeto a verificación (opción 1) y la probabilidad de que este no suceda (opción 0), se interpreta como la preferencia o ventaja de lo primero respecto a lo segundo.

Con base en la teoría subjetiva del valor, en este trabajo se espera encontrar una relación no lineal entre las variables explicativas y la probabilidad de ocurrencia del evento relevante, por la cual las variaciones en la probabilidad provocadas por cambios en estas variables dependerían de su nivel original. Esta relación no lineal sugeriría que las razones por las que el promotor de un emprendimiento sin establecimiento decide montar un local para la venta de sus productos o prestación de sus servicios, no son necesariamente las mismas que hacen

\footnotetext{
${ }^{3}$ Para efectos de este ejercicio se entiende que un proyecto microempresarial será de mayor tamaño cuanto mayor sea su número de establecimientos, siendo desestimadas como medidas directas de crecimiento opciones tales como el número de empleados, la tasa de rentabilidad o el nivel de activos (Fuertes-Callén y Cuellar-Fernández, 2019).
} 
que un emprendedor con un establecimiento resuelva poner una sucursal. En este sentido, las transiciones permiten distinguir el efecto de las variables explicativas en, por un lado, la probable salida del autoempleo (vinculada a la puesta en marcha del primer establecimiento), y por el otro, la consolidación de la microempresa (con la apertura del segundo establecimiento).

Para resaltar estas diferencias subyacentes en la variable dependiente, en este trabajo se ha optado por estimar dos modelos Logit binarios (cada uno con dos categorías: una inferior y otra superior) como método de análisis estadístico. En el primero de estos, la variable dependiente refleja el tránsito o no, de estar "sin establecimiento" a contar con "un establecimiento" durante el último año (caso 0-1). El segundo caso se refiere al paso o no, de tener "un establecimiento" a contar con "dos establecimientos" durante el último año (caso 1-2). Se asume que este tratamiento permite diferenciar la naturaleza de los emprendimientos.

Los datos utilizados provienen de la Encuesta Nacional de Ingresos y Gastos de los Hogares (ENIGH) 2016, que recoge bienalmente información individual acerca de las características socioeconómicas de los miembros del hogar, incluyendo las fuentes de ingresos -tanto formales como informales- y el destino del gasto (INEGI 2016c). Esta encuesta, que es levantada por el INEGI, tiene un diseño de corte transversal y cuenta con representatividad nacional. La ENIGH reserva uno de sus cuestionarios a la información de los negocios del hogar, cuyo tamaño de muestra en el año de referencia fue de 5,294 observaciones. Aunque esta constituye la fuente de información básica, la muestra final utilizada en esta investigación estuvo compuesta de 1,860 observaciones, al ceñirse a los casos de los sectores económicos industrial, comercial y de servicios y a aquellos que no contaban con acta constitutiva y registro ante notario, entidad federativa o autoridad hacendaria (emprendimientos de tipo informal).

Cabe destacar que el tamaño medio de hogar de la muestra es de 3.9 personas, mientras que la edad media del responsable del negocio es de 47.7 años. Además, aunque los perceptores de ingreso por hogar son en promedio 2.6 personas, los integrantes ocupados en este son 1.5 personas. No se debe pasar por alto que al menos el $15.1 \%$ de las remuneraciones por trabajo en México tiene su origen en actividades no subordinadas como las de los negocios de hogar. Con base en el número de observaciones, la participación de las entidades federativas en la muestra va desde el 5.0\% para el caso de Baja California, hasta el 2.5\% para la Ciudad de México, mientras que el peso de las zonas urbanas va desde el $98.9 \%$ en la Ciudad de México, hasta el $45.6 \%$ en Baja California.

En cuanto a las variables que pueden tener relación, directa o indirecta, con el crecimiento empresaria, Forth y Bryson (2018), encuentran que la productividad y la constancia en la operación pueden ser útiles para explicar la evolución de la microempresa. Rivera (2018), 
por su parte, señala que la asociatividad, el sector de actividad, el número de locales, la conformación de la plantilla y el nivel de ganancias pueden ayudar a explicar la heterogeneidad en la conformación de los emprendimientos en México.

La Tabla 1 presenta las variables empleadas en la especificación de los modelos estadísticos de este trabajo, las cuales han sido seleccionadas con base en la literatura y la disponibilidad de información.

Tabla 1

Variables utilizadas en el análisis estadístico

\begin{tabular}{|c|c|c|c|}
\hline Tipo & Nombre & Definición & Formas de respuesta** \\
\hline \multirow[t]{5}{*}{-} & $\begin{array}{l}\text { Número de estableci- } \\
\text { mientos* }\end{array}$ & $\begin{array}{l}\text { Sucursales dedicadas a la misma actividad } \\
\text { comercial o productiva, relacionadas con un } \\
\text { mismo propietario. }\end{array}$ & $\begin{array}{l}\text { De } 0 \text { a } 2 \text { (según el caso } \\
\text { de que se trate). }\end{array}$ \\
\hline & $\begin{array}{l}\text { Tipo de actividad econó- } \\
\text { mica }\end{array}$ & $\begin{array}{l}\text { Aquello que define las acciones y procesos } \\
\text { que caracterizan la organización del trabajo } \\
\text { orientado a la producción y comercializa- } \\
\text { ción de bienes y servicios. }\end{array}$ & $\begin{array}{l}\text { 1: Industrial, } \\
\text { 2: Comercial, } \\
\text { 3: De servicios. }\end{array}$ \\
\hline & Número de socios & $\begin{array}{l}\text { Personas que aportaron capital inicial o que } \\
\text { tienen injerencia en las decisiones y a las } \\
\text { que hay que entregar parte de las ganancias. }\end{array}$ & De 0 a 10. \\
\hline & Número de empleados & $\begin{array}{l}\text { Trabajadores (remunerados o no) bajo las } \\
\text { órdenes de un patrón. }\end{array}$ & De 0 a 10. \\
\hline & $\begin{array}{l}\text { Porcentaje de empleados } \\
\text { hombres }\end{array}$ & $\begin{array}{l}\text { Conjunto de trabajadores del sexo masculi- } \\
\text { no como proporción del total. }\end{array}$ & De 0 a 100 . \\
\hline \multirow[t]{4}{*}{ De gestión } & $\begin{array}{l}\text { Tipo de empleo que } \\
\text { representa el emprendi- } \\
\text { miento }\end{array}$ & $\begin{array}{l}\text { Prioridad -en términos de ingresos- que re- } \\
\text { presenta la actividad emprendedora. }\end{array}$ & $\begin{array}{l}\text { 1: Primario, } \\
\text { 2: Secundario. }\end{array}$ \\
\hline & $\begin{array}{l}\text { Hacer uso de servicios } \\
\text { contables }\end{array}$ & $\begin{array}{l}\text { Hecho de apoyarse de servicios externos } \\
\text { para llevar el control financiero y contable. }\end{array}$ & 1: No, 2: Sí. \\
\hline & $\begin{array}{l}\text { Tomar bienes para auto- } \\
\text { consumo }\end{array}$ & $\begin{array}{l}\text { Hecho de consumir, por parte del emprende- } \\
\text { dor o su familia, bienes o servicios del em- } \\
\text { prendimiento por los que no se paga ningún } \\
\text { valor. }\end{array}$ & 1: No, 2: Sí. \\
\hline & $\begin{array}{l}\text { Porcentaje del año en } \\
\text { operación }\end{array}$ & $\begin{array}{l}\text { Proporción de los meses del año en los que } \\
\text { se desarrolla la actividad (con base en el úl- } \\
\text { timo año). }\end{array}$ & $\begin{array}{l}\text { De } 0 \text { a } 100 \text {, en interva- } \\
\text { los de } 8.33 \text {. }\end{array}$ \\
\hline
\end{tabular}




\begin{tabular}{|c|c|c|c|}
\hline \multirow{3}{*}{$\begin{array}{l}\text { De } \\
\text { resultados }\end{array}$} & Productividad $* * *$ & (ingresos-gastos) / número de empleados. & $\begin{array}{l}\text { De } \$-1,083 \\
\text { a } \$ 5,149 .\end{array}$ \\
\hline & $\begin{array}{l}\text { Variabilidad de las ven- } \\
\text { tas }\end{array}$ & $\begin{array}{l}\text { Desviaciones estándar respecto a la media, } \\
\text { necesarias para cubrir el rango completo de } \\
\text { ventas. }\end{array}$ & De 0 a 2.45 . \\
\hline & Rentabilidad***** & $100 \times$ (ingresos-gastos) / ingresos. & De -200 a 100. \\
\hline
\end{tabular}

Fuente: Elaboración propia con información de la INEGI (2016c).

Según el papel que juegan en el proceso de operación, las variables han sido clasificadas como: de gestión y de resultados, lo que facilita el reconocimiento de un entorno favorable para la expansión, al diferenciar el comportamiento del microempresario del desempeño de su proyecto. Las variables de gestión estarían asociadas al empeño, mientras que las de resultados lo harían al desempeño. Aunque se reconoce que la gestión puede incidir en los resultados (Rivera, 2018), se considera que esto no ocurre de forma exclusiva ni necesariamente directa. Como se ha dicho ya, es probable que otras variables del lado de la demanda como el ingreso disponible, el entorno competitivo o las preferencias de los consumidores incidan tanto en el desempeño de la microempresa como en su propia existencia. No obstante, la inclusión de dichas variables requeriría de un trabajo de campo que excede las posibilidades de esta investigación, por lo que se suponen constantes.

Por último, se implementan dos filtros para elevar la confianza en la identificación de cambios en la escala de negocios de las unidades de análisis. Por una parte, se incluyen solo los emprendimientos de entre cero y dos establecimientos en el afán de homogeneizar las muestras y caracterizar mejor los comportamientos; por la otra, se consideran únicamente los emprendimientos con al menos un año de operación para reducir la posibilidad de inclusión de proyectos en etapa de creación (cuyo cambio en la escala de negocios no corresponde a un proceso de expansión). Así, la submuestra del caso 0-1, se compone de los emprendimientos que en el último año no habían montado un establecimiento y de aquellos con evidencia de haberlo hecho; mientras que, la del caso 1-2, se conforma de los emprendimientos que durante el último año se habían mantenido con un establecimiento y de aquellos donde había evidencia de que habían llegado a dos. 


\section{Resultados}

Con la intención de caracterizar el sector microempresarial por medio de estadísticos básicos se hace una revisión exploratoria de la base de datos (antes de aplicar los filtros presentados en la sección anterior). De dicho análisis se obtiene que el 63.2\% de los negocios de hogar tiene solo un establecimiento y únicamente el $1.9 \%$ tiene más de uno (en cuyo el caso el sector predominante es el de los servicios); sin embargo, el 34.9\% no cuenta con establecimiento o área exclusiva para realizar sus actividades de comercialización. Asimismo, el 62.1\% de los negocios no cuenta con empleados y el $25.2 \%$ solo tiene uno; únicamente el $12.7 \%$ tiene más de un empleado. Lo anterior estaría relacionado con el hecho de que apenas el 54.3\% de la muestra declaró llevar a cabo estas tareas en su domicilio particular, en el de familiares o en puestos fijos en vía pública, mientras que el resto se apoyaba del domicilio de los clientes, de puestos semifijos o en la práctica del ambulantaje.

En cuanto al sector microempresarial de actividad, con una representatividad del $51.5 \%$, el preponderante es el comercial, seguido del de servicios con 38.3\%, mientras que el industrial, con una participación de $10.2 \%$, está en tercer sitio. Además, el $85.1 \%$ de los emprendimientos lleva más de un año de operación, y de estos, $91.3 \%$ presenta continuidad en el desarrollo

de la actividad; el resto es de naturaleza estacional o afronta algún problema operativo (o un elevado costo de oportunidad en términos de ingreso) que hace inconveniente o impide a su propietario ser constante en la operación. Al respecto, el tiempo en operación alcanzado, al ser prueba de persistencia y condición para adquirir experiencia, juega un papel importante en la proyección de la vida futura del emprendimiento (por ejemplo, esta estimación es muy distinta de haberse superado o no el punto de equilibrio económico).

El porcentaje de personal masculino dentro de los emprendimientos con empleados varía con el tamaño de negocios: en aquellos que no tenían establecimiento era de 56.9\%, en los que tenían un establecimiento, de $52.5 \%$, y en los que tenían más de un local, 38.2\%. Se advierte así que la mayor presencia relativa de mujeres trabajadoras en negocios de hogar aumenta con el tamaño de estos. También, alrededor de la mitad de la plantilla de aquellas microempresas con empleados y a lo sumo un local comercial no tenía un salario fijo. En este sentido, Aguilar, Mungaray y Ramírez (2014) aseveran que la falta de salario fijo en microempresas es mayor cuando los empleados son parte de la familia, todavía más cuando estos familiares son mujeres.

Adicionalmente se observa que, a mayor número de establecimientos, mayor es la probabilidad de que se cuente con servicios contables y de que se entregue comprobante fiscal de 
venta. En 2015, el $96.7 \%$ y $98.7 \%$ de los emprendimientos sin local no utilizaban servicios contables externos y no entregaban prueba de venta, respectivamente. Los indicadores se reducían a $80.4 \%$ y $92.1 \%$ cuando se contaba con un establecimiento exclusivo para la actividad, y llegaban a $54.8 \%$ y $78.4 \%$ de contarse con dos establecimientos. Se destaca que en 2018 la ausencia de apoyo contable externo en microempresas formales era inferior a 50.0\%, aunque una de cada cinco seguía apoyándose del cuadernillo de notas como base del registro de sus operaciones (INEGI, 2016a; INEGI, 2019).

El autoconsumo (tomar artículos o disfrutar de servicios del negocio sin que se cubra el costo de producción o se registre la operación) favorece la liquidez de corto plazo, pero denota una deficiencia en el control de la gestión de la microempresa que pone en riesgo su permanencia en el mercado. Con relación a este comportamiento, de no contarse con establecimiento, el autoconsumo había ocurrido en el último mes en el $49.8 \%$ de los emprendimientos; cuando se tenía un punto de venta, se daba en el $65.4 \%$, pero cuando se tenían dos, el indicador disminuía a 35.6\%. Esto sugiere un mayor "descontrol" cuando el emprendimiento tiene solo un establecimiento, lo que posiblemente esté relacionado con la incorporación de los primeros empleados, en muchos casos, familiares sin pago. Se destaca que, en el $54.2 \%$ de las microempresas donde se presentaba autoconsumo, el valor de lo tomado oscilaba entre $\$ 6$ y $\$ 60$, iba de $\$ 60$ a $\$ 600$ en el $34 \%$ de los casos, e incluso, en el $12.3 \%$ de estos, se tomaron bienes cuyo valor estaba entre $\$ 600$ y $\$ 900$, lo cual supera el nivel medio de ingresos de muchas de las microempresas estudiadas, como se verá más adelante.

En materia de asociatividad se encontró que el $97.1 \%$ de los microempresarios informales en México no tiene socios distintos a los miembros del hogar; cuando los posee, tanto estos como las sociedades resultantes, tienen alta probabilidad de ser también informales. Controlando por la dimensión de negocios, solo $2.8 \%$ de los proyectos sin local presenta socios; cuando tienen un local el indicador sube a 3.6\%; mientras que presenta sociedad en su organización el $3.7 \%$ de los emprendimientos con dos locales. Así, no se aprecian cambios significativos en la estructura asociativa de los emprendimientos según el número de locales; tampoco es clara la incidencia de dicha variable sobre la calidad de la gestión.

En cuanto a resultados económico-financieros, se encuentra que el $78.8 \%$ de estas microempresas obtiene ingresos mensuales por ventas de hasta $\$ 600^{4}$; en el $18.5 \%$ de los casos estos ingresos van de $\$ 600$ a $\$ 3,000$; el porcentaje restante presenta niveles superiores a $\$ 3,000$ en el periodo. En lo referente a los gastos, el $86.2 \%$ requiere mensualmente de hasta $\$ 600$ para la operación (que es un porcentaje mayor al de las microempresas con

\footnotetext{
${ }^{4}$ Los valores monetarios están expresados en dólares americanos al tipo de cambio vigente en mayo de 2018 .
} 
ingresos de hasta esa cuantía) y en el $12.1 \%$ de los casos los gastos van de $\$ 600$ a $\$ 3,000$; el resto supera ese umbral.

Asimismo, destaca la relación positiva entre el indicador de productividad (la utilidad por empleado) y el número de establecimientos, iniciando en \$217.2 cuando no se tiene local, hasta alcanzar \$421.3 para el caso en el que se cuenta con dos locales. No obstante, el indicador de rentabilidad (el margen de utilidad) se muestra decreciente con el número de establecimientos, partiendo de $36.9 \%$ cuando no se tiene local, hasta llegar a $19.0 \%$ cuando se cuenta con dos. Finalmente, la variabilidad de las ventas, que es un indicador de incertidumbre, también disminuye con el número de establecimientos. Lo anterior, aunque sugiere la necesidad de mayor estabilidad para poder crecer, también podría significar que los retornos a la inversión asociados a una expansión podrían verse comprometidos, lo que puede constituir un obstáculo al crecimiento. De esta forma, los resultados anteriores apoyarían solo parcialmente las tesis tanto de Federico y Capelleras (2015) como de Fuertes-Callén y Cuellar-Fernández (2019), que aducen que el crecimiento suele favorecer la mejora de los indicadores de resultados.

La exploración de las submuestras de los casos tratados (0-1 y 1-2), revela que, en ambas, las observaciones asociadas a la categoría inferior presentan una menor proporción de la actividad microempresarial como primera fuente de ingreso. Además, la presencia de trabajadores sin pago mostró niveles menores en la forma de respuesta superior para el caso 1-2 pero se mantuvo relativamente constante en el caso 0-1. En términos generales, estos resultados responden a la lógica económica.

La Tabla 2 muestra los resultados obtenidos (tanto a nivel de coeficientes como de valores medios) al regresar las especificaciones Logit para cada una de las submuestras (casos 0-1 y 1-2) con el objetivo de identificar las relaciones existentes entre las variables. Dado que los signos de los coeficientes del Logit reflejan la relación entre variables independientes y dependiente pero no de forma directa los efectos marginales (elasticidades) asociados a la relación entre variables, se agregan los coeficientes de pendiente parcial con el fin de favorecer la interpretación. 
Tabla 2

Resultado de las estimaciones Logit para dos casos de expansión microempresarial

\begin{tabular}{|c|c|c|c|c|c|}
\hline \multirow[t]{2}{*}{ Tipo } & \multirow[t]{2}{*}{ Variables independientes } & \multicolumn{2}{|c|}{$\begin{array}{c}\text { Coeficiente } \\
\text { (Error estándar) }\end{array}$} & \multicolumn{2}{|c|}{$\begin{array}{c}\text { Media } \\
\text { (Pendiente parcial**) }\end{array}$} \\
\hline & & Caso 0-1 & Caso 1-2 & Caso 0-1 & Caso 1-2 \\
\hline & $($ Conctonta)* $\%$ & 3.1805 & -0.0751 & & \\
\hline & (comstame) & $(0.7827)$ & $(1.3688)$ & - & - \\
\hline \multirow{16}{*}{ De gestión } & Tipo de actividad & 0.3384 & & 2.0890 & \\
\hline & económica & $(0.0731)$ & - & $(0.0835)$ & - \\
\hline & Número de socine & -0.2692 & & 0.0731 & 列 \\
\hline & Numero de socios & $(0.1599)$ & - & $(-0.0664)$ & - \\
\hline & & 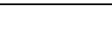 & 0.7980 & 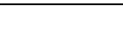 & 1.7900 \\
\hline & Numero de empleados & - & $(0.0942)$ & - & $(0.0422)$ \\
\hline & Porcentaje de empleados & - & 0.0072 & & 49.3907 \\
\hline & hombres & - & $(0.0033)$ & - & $(0.0004)$ \\
\hline & Tipo de empleo que repre- & & -1.5736 & & 1.1510 \\
\hline & senta el emprendimiento & - & $(0.6556)$ & - & $(-0.0833)$ \\
\hline & Hacer uso de servicios & 2.2842 & 1.3317 & 1.8750 & 1.8111 \\
\hline & contables & $(0.2784)$ & $(0.2769)$ & $(-0.5634)$ & $(-0.0705)$ \\
\hline & Tomar bienes para auto- & 0.2604 & -1.0079 & 1.4391 & 1.3693 \\
\hline & consumo & $(0.1152)$ & $(0.2663)$ & $(-0.0643)$ & $(0.0533)$ \\
\hline & Porcentaje del año en & 0.0114 & -0.0178 & 95.7420 & 98.1802 \\
\hline & operación & $(0.0049)$ & $(0.0089)$ & $(0.0028)$ & $(0.0009)$ \\
\hline \multirow{12}{*}{ De resultados } & Deodurtivided 12 & 0.0130 & & 294.5123 & \\
\hline & Productividad & $(0.0002)$ & - & $(0.0003)$ & - \\
\hline & Variabilidad de las ventas & -0.4818 & - & 0.2263 & - \\
\hline & Varrabindad de las ventas & $(0.1843)$ & - & $(-0.1188)$ & - \\
\hline & Rentabilidad & -0.0116 & - & 28.9814 & - \\
\hline & Kentabindad & $(0.0017)$ & - & $(-0.0029)$ & - \\
\hline & $R^{2}$ de McFadden & 0.1546 & 0.3081 & - & - \\
\hline & Porcentaje de aciertos & 66.9 & 90.9 & - & - \\
\hline & Criterio de Akaike & $1,889.2$ & 430.2 & - & - \\
\hline & Test Hosmer-Lemeshow & 7,935 & 6,063 & & \\
\hline & $x^{2}$ (significancia) & $(0.115)$ & $(0.469)$ & - & - \\
\hline & $n$ & 1,811 & 704 & - & - \\
\hline
\end{tabular}

La variable dependiente refleja la ocurrencia o no del incremento en un establecimiento. Todas las variables son significativas al menos al $10 \%$ excepto la constante del caso $1-2 . *$ La constante funciona como variable de ajuste contribuyendo a reducir el nivel de error del modelo. ** Cambio en la variable dependiente correspondiente una unidad en la regresora manteniendo constante el resto.

Fuente: Elaboración propia con base en INEGI (2016c). Para el análisis estadístico se utilizó SPSS 24.0. 
Las estimaciones prueban la existencia de relaciones entre una serie de atributos en las microempresas (representados tanto por variables de gestión como de resultados), sus condiciones iniciales y el número de establecimientos que estas deciden tener. Dichas relaciones sugieren una diferenciación estructural en el contexto de la decisión de expansión. Entre las variables de gestión que propician de forma exclusiva el paso a la apertura del primer establecimiento están el tipo de actividad económica y el número de socios; específicamente se trataría de las actividades comerciales o de servicios y de mantener un reducido número de socios, lo que no es incompatible con la gran importancia de las redes sociales en el desarrollo empresarial aludida por Berge et al. (2015) y Thapa (2015). En contraste, el aumento en la plantilla, el incremento de la fuerza laboral masculina y la prioridad del proyecto en la actividad laboral del emprendedor estarían exclusivamente asociadas a poner un segundo establecimiento.

Otras variables como el uso de servicios contables - una de las principales condiciones necesarias para el crecimiento de acuerdo con lo encontrado en esta investigación-, el nivel de autoconsumo y la constancia en la operación se presentan como significativos en ambas regresiones. No obstante, en estas dos últimas variables, los signos difieren entre los casos. La interpretación de estos resultados se presenta a continuación.

Aun sin entrar en temas de causalidad, es posible conjeturar que, el uso de servicios contables favorece la transición hacia una configuración empresarial más robusta. El impacto de este indicador es mayor conforme más pequeño sea el emprendimiento. Paralelamente, la práctica del autoconsumo afecta negativamente la probabilidad de abrir un segundo local, pero es compatible con el establecimiento del primero. Pudiera ser que, en los emprendimientos con organización muy básica, el autoconsumo llegue a formar parte de las retribuciones al trabajo de los empleados; esta situación deja de tener sentido conforme el emprendimiento crece, haciendo necesaria la implementación de más controles.

Mantener continuidad en el desarrollo de la actividad microempresarial incrementa la propensión a que el emprendimiento tenga un local, pero lo aleja de la posibilidad de tener dos, lo que constituye un resultado no esperado. Es probable que esto tenga que ver con la flexibilidad asociada al sector servicios, que presenta la mayor participación en los proyectos con más de un establecimiento. En cuanto al efecto del tiempo en operación en el crecimiento de un proyecto, los resultados de la literatura están en función de las características de la microempresa, lo cual se corrobora en este trabajo. Con todo, parece que la probabilidad de expandirse se hace decreciente a partir de tener algunos años en operación (Nichter y Goldmark, 2005).

Se observa que solo el caso 0-1 presentó significancia estadística en las variables relacionadas con los resultados. Esta va en el sentido de que mayor productividad y estabilidad 
en el nivel de ventas contribuyen al establecimiento del primer local; no obstante, el comportamiento de la rentabilidad es inverso. Una posible explicación de este resultado aparentemente contraintuitivo es que la frecuente indivisibilidad entre la propiedad y el control en este tipo de empresas (en el sentido de Allinson et al., 2013), contribuye a que altos niveles de rentabilidad, si son alcanzados en corto tiempo, condenan la evolución de la actividad en términos de su dimensión de negocios, más allá de que estos niveles superen con creces las necesidades del umbral de subsistencia. Lo anterior también muestra una correspondencia tangencial con la evidencia empírica presentada por Federico y Capellares (2015) y Monge y Torres (2015) en el sentido de que la relación que puede haber entre crecimiento y beneficios es más directa para las empresas más jóvenes, aunque esto no siempre se refleje en una evolución en la estratificación empresarial.

Por otra parte, variables tales como el lugar donde se realiza la actividad, la proporción de empleados familiares o el número de dependientes económicos, no resultaron estadísticamente significativas en ninguna de las regresiones, por lo que fueron excluidas de la especificación final, mientras que variables como la entrega de comprobantes de venta y la presencia de trabajadores sin pago, fueron descartadas del análisis para evitar problemas de multicolinealidad.

Respecto a las pruebas de bondad de ajuste (ver Tabla 2), los modelos se evaluaron por el criterio de Akaike que selecciona la especificación con el error cuadrado más pequeño. Además, se calcularon los $R^{2}$ de McFadden que aunque no se acercan demasiado a la unidad, como es de esperar en los modelos no lineales y los estimados con base en encuestas de hogares, es posible asegurar que el ajuste es aceptable dada la estructura de los datos con base en la significancia de la prueba de Hosmer-Lemeshow.

Adicionalmente, las tablas 3 y 4 muestran, con apoyo de los resultados dela Tabla 2, las probabilidades de pasar, respectivamente, de 0 a 1 y de 1 a 2 establecimientos en el rango relevante de cada variable regresora cuando el resto toma su valor medio. Para el caso 0-1 (Tabla 3), se encuentra que utilizar servicios contables, apoyarse del autoconsumo (cuidado de la liquidez), incrementar la productividad, no contar con socios (reducción de riesgo de comportamientos oportunistas ante la falta de contratos), ser constante en la operación (en línea con Berrone et al., 2014) y en el nivel de ventas, y no presentar incrementos abruptos en la rentabilidad (reducción del riesgo de gasto en bienes duraderos para el hogar, por ejemplo) estarían asociados a una probabilidad de $94.7 \%$ de que un emprendimiento establezca su primer local comercial en el corto plazo. 
Tabla 3

Probabilidades de pasar de 0 a 1 establecimiento en el límite del rango relevante de las variables regresoras

\begin{tabular}{llclc}
\hline $\begin{array}{l}\text { Variables que se } \\
\text { evalúan en el límite }\end{array}$ & Límite inferior del rango & Prob. (\%) & Límite superior del rango & Prob. (\%) \\
\hline & Un socio & 49.7 & Ningún socio & 56.4 \\
& No servicios de contador & 48.8 & Sí servicios de contador & 90.3 \\
Cada variable, & No autoconsumo & 52.2 & Sí autoconsumo & 58.7 \\
evaluando al resto & Medio año en operación & 42.9 & Un año en operación & 57.1 \\
en la media & Actividad comercial & 55.1 & Actividad de servicios & 63.3 \\
& Productividad de \$200 & 52.9 & Productividad de \$250 & 54.5 \\
& Baja variabilidad en ventas & 46.6 & Nula variabilidad en ventas & 58.5 \\
Todas las variables & Rentabilidad de 35\% & 54.2 & Rentabilidad de 20\% & 58.4 \\
\hline
\end{tabular}

Fuente: Elaboración propia con base en INEGI (2016c). Para el análisis estadístico se utilizó SPSS 24.0.

Para el caso 1-2, la Tabla 4 muestra que utilizar servicios de contador, no autoconsumir bienes de la empresa (acorde con lo señalado por Hayes et al., 2015, en lo referente al manejo de insumos), incrementar la proporción de hombres en la plantilla (inequidad de género), ser la actividad laboral prioritaria del emprendedor, incrementar el número de empleados y ser más flexible en la operación (por ejemplo, por la tercerización de la economía), se pueden asociar a una probabilidad de $61.3 \%$ de que el emprendedor establezca un segundo local comercial en el corto plazo.

\section{Tabla 4}

Probabilidades de pasar de 1 a 2 establecimientos en el límite del rango relevante de las variables regresoras

\begin{tabular}{llclc}
\hline $\begin{array}{l}\text { Variables que se } \\
\text { evalúan en el límite }\end{array}$ & Límite inferior del rango & Prob. (\%) & Límite superior del rango & Prob. (\%) \\
& & & & \\
\hline & Un empleado & 2.9 & Dos empleados & 6.2 \\
Cada variable, & 50\% de empleados hombres & 5.3 & $100 \%$ de empleados hombres & 7.5 \\
evaluando al resto & Se trata de empleo secundario & 1.5 & Se trata de empleo primario & 6.6 \\
en la media & No servicios de contador & 4.2 & Sí servicios de contador & 14.2 \\
& Sí autoconsumo & 3.7 & No autoconsumo & 9.6 \\
Todas las variables & Un año en operación & 5.1 & Medio año en operación & 11.7 \\
\hline
\end{tabular}

Fuente: Elaboración propia con base en INEGI (2016c). Para el análisis estadístico se utilizó SPSS 24.0. 
Finalmente, las probabilidades de pasar a la categoría superior, calculadas en los valores medios de las variables, son de 55.9\% para el caso 0-1 y de 5.3\% para el caso 1-2. La divergencia entre estas probabilidades junto con la estadística exploratoria y los resultados del análisis de significancia y signos, en ambos modelos, constituye suficiente evidencia como para no rechazar la hipótesis de que las posibilidades de crecimiento de la microempresa informal, aunque puedan estar asociadas a sus resultados, también lo estaría a sus condiciones iniciales, estructura y visión del promotor.

\section{Conclusiones}

En los últimos años se ha visto una clara tendencia en el accionar de los gobiernos latinoamericanos hacia el fortalecimiento de los programas de financiamiento y asistencia técnica que propician la creación, desarrollo y consolidación de microempresas. El objetivo de lo anterior es que estas acciones puedan servir como mecanismo de despresurización del mercado laboral y dinamización de la economía. Sin embargo, la realidad es que, más allá de que los emprendimientos puedan tener buenos resultados económico-financieros de corto plazo, son muy pocos los que terminan por expandirse y dar muestra de algunos de los beneficios que se le suponen, tales como la generación de empleo, el pago de impuestos y cargas sociales, la participación en el desarrollo local y la promoción de la competencia económica, entre otros.

Con información de la INEGI (2016c) y mediante la estimación de modelos Logit, este trabajo estudia, para los emprendimientos de bajo valor agregado de tipo informal, la incidencia de distintas variables relacionadas con su gestión y resultados, con la intención de aportar evidencia empírica que contribuya a identificar el efecto en las decisiones de expansión de, por una parte, la gestión y empeño de los propietarios y, por la otra, el desempeño del emprendimiento.

Aunque se pueden enumerar muchas buenas razones para querer hacer que un emprendimiento crezca, la sola administración de este proceso es todo un reto que no siempre entra en los planes de aquellos que en algún momento iniciaron un proyecto microempresarial. En este trabajo se pudo constatar que, establecer el primer local comercial y evolucionar hacia un segundo establecimiento (muchas veces entendidos como elementos secuenciales dentro de un proceso inercial de expansión del negocio), son decisiones estructural y motivacionalmente diferentes.

Aunque muchos elementos característicos de la microempresa informal no son sensibles al tiempo en operación (lo que en parte evidencia las limitaciones de expansión que presentan), 
existen factores relacionados con la gestión y el empeño, y no solo aquellos relacionados con los resultados, capaces de ofrecer una explicación acerca de este proceso de crecimiento. En todo caso se debe tener en cuenta que la visión de futuro en estos emprendimientos parece estar dirigida en buena medida por necesidades económicas de corto plazo, y delimitada por aspectos culturales y una diversidad de prejuicios acerca de lo que representan la operación del negocio, la formalidad y las relaciones sociales e institucionales.

No se puede concluir que el principal detonador de la expansión microempresarial sea un buen desempeño o el tiempo en operación. De hecho, las relaciones de estas variables con el crecimiento son débiles o nulas, además de estructuralmente diferentes según la naturaleza y dimensión del emprendimiento. Lo anterior podría tener relación con el contraste entre emprendimientos de subsistencia y de acumulación, por el que serían estos últimos, los que presentan un mayor potencial de crecimiento, a diferencia de las microempresas de subsistencia cuyos objetivos se dirigen a la búsqueda de la seguridad familiar, o la maximización del ingreso o de la capacidad instalada.

Autores como Rivera (2018) creen que los emprendimientos informales, en cuyo crecimiento, el empeño del propietario juega un papel relevante como se ha mostrado en este trabajo (reforzando el punto de vista de trabajos como el de Forth y Bryson, [2018]), deben ser apoyados a través de políticas de largo plazo que consideren la provisión de créditos, e incluso la asistencia social. De cualquier forma, lo anterior deberá darse en condiciones apropiadas a la escala de negocios y a la formación y capacitación recibidas, y acompañarse de un entorno que favorezca una mayor dedicación al emprendimiento con miras a su profesionalización.

El efecto de estas políticas en el bienestar dependerá de la claridad que tengan los responsables de su diseño e implementación acera del tipo de éxito que quieren promover a través de intervenciones (Garoma, 2012), lo cual pasa por la identificación de las motivaciones de este sector y por el reconocimiento de que, si se busca formalizar, consolidar y expandir los emprendimientos que un día nacieron como informales (y posiblemente como autoempleos), el impulsar el buen desempeño económico, aunque conveniente, podría no ser suficiente.

Será necesario explorar más profundamente la naturaleza y los fines de estos emprendimientos, y así evitar la tentación de caer en generalizaciones que lleven a conclusiones imprecisas acerca de su verdadero potencial de expansión. Trabajos futuros en esta línea de investigación deben considerar la inclusión de variables que sirvan de ajuste del modelo tales como el sexo, el estatus de formalidad y el nivel de ingreso por zona geográfica, además de otras que reflejen de forma más directa el resultado del empeño del emprendedor, las cuales, por distintas limitaciones que fueron surgiendo a lo largo del estudio, no fue posible incorporar. 


\section{Referencias}

Aguilar, J. G., Mungaray, A. B., \& Ramírez, M. (2014). Género y autoempleo en México durante la recesión de 2008-2009: un estudio de flujos laborales. Papeles de Población, 20 (80), 145-191.

Allen, I. E., Elam, A., Langowitz, N. \& Dean, M. (2008). Global Entrepreneurship Monitor, 2007 Report on Women and Entrepreneurship. The Center for Women's Leadership at Babson College.

Allinson, G., Braidford, P., Houston, M. \& Stone, I. (2013). Understanding growth in microbusinesses. BIS Research Paper 114. Department for Business Innovation \& Skills. Disponible en: http://dro.dur.ac.uk/14736/1/14736. pdf?DDD2+dbr1gfa+d700tmt+dul4eg (Consultado: 11/7/2019)

Berge, L., Bjorvatn, K. \& Tungodden, B. (2015). Human and financial capital for microenterprise development: Evidence from a field and lab experiment. Management Science, 61 (4), 707-722. https://doi.org/10.1287/mnsc.2014.1933

Berner, E., Gomez, G. \& Knorringa, P. (2012). Helping a large number of people become a little less poor: The logic of survival entrepreneurs. The European Journal of Development Research, 24 (3), 382-396. https://doi. org/10.1057/ejdr.2011.61

Berrone, P., Gertel, H., Giuliodori, R., Leandra, B. \& Meiners, E. (2014). Determinants of performance in microenterprises: Preliminary evidence from Argentina. Journal of Small Business Management, 52 (3), 477-500. https://doi.org/10.1111/jsbm.12045

Cruz, M., López, E., Cruz, R. \& Meneses, G. (2016). ¿Por qué no crecen las micro y pequeñas empresas en México? En R. Paredes, N. Peña e I. Vacio (Coords.) La micro y pequeña empresa: un análisis desde la perspectiva económico-administrativa (329-339), T-I -CECORFAN-San Juan del Río, Querétaro.

Dawson, C. \& Henley, A. (2012). "Push" versus "pull” entrepreneurship: An ambiguous distinction? International JournalofEntrepreneurial Behaviour \& Research.18(6), 697-719. https://doi.org/10.1108/13552551211268139

Dini, M. \& Stumpo, G. (coords.) (2018), MiPyMEs en América Latina: un frágil desempeño y nuevos desafíos para las políticas de fomento. Documentos de Proyectos (LC/TS.2018/75), Santiago, Comisión Económica para América Latina y el Caribe (CEPAL).

Docquier, F., Müller, T. \& Naval, J. (2017), Informality and long-run growth. The Scandinavian Journal of Economics, 119 (4), 1040-1085. https://doi.org/10.1111/sjoe.12185

Federico, J. \& Capelleras, J. (2015). The heterogeneous dynamics between growth and profits: the case of young firms. Small Business Economics, 44 (2), 231-253. https://doi.org/10.1007/s11187-014-9598-9

Fuertes-Callén, Y. \& Cuellar-Fernández, B. (2019). Inter-relationship between firm growth and profitability in a context of economic crisis, Journal of Business Economics and Management, 20 (1), 86-106. https://doi. org/10.3846/jbem.2019.6928

Forth, J. \& Bryson, A. (2018). The impact of management practices on SME performance. IZA Discussion Paper No. 11399. Social Science Research Network (SSRN): Disponible en: https://ssrn.com/abstract=3153363 (Consultado: 4/9/2019)

Garoma, B. (2012). Determinants of microenterprise success in the urban informal sector of Addis Ababa: A multidimensional analysis (Tesis de doctorado). Erasmus University Rotterdam, Países Bajos.

Hayes, J., Chawla, S. \& Kathawala, Y. (2015). A comparative study of problems encountered in the development of small businesses in the U.S. and Mexico. The Journal of Developing Areas, 49(3), 395-406. https://doi. org/10.1353/jda.2015.0175

Holm, H., Opper, S. \& Nee, V. (2013). Entrepreneurs under uncertainty: An economic experiment in China. Management Science, 59 (7), 1671-1687. https://doi.org/10.1287/mnsc.1120.1670

INEGI (2016a). Encuesta nacional sobre productividad y competitividad de las micro, pequeñas y medianas Empresas (ENAPROCE). Instituto Nacional de Estadística y Geografía. Aguascalientes, México.

INEGI (2016b). Boletín de Prensa Núm. 285/16. Instituto Nacional de Estadística y Geografía. Disponible en: http://www.inegi.org.mx/saladeprensa/boletines/2016/especiales/especiales2016_07_02.pdf (Consultado: 8/4/2019) 
INEGI (2016c). Encuesta Nacional de Ingresos y Gastos de los Hogares (ENIGH). Instituto Nacional de Estadística y Geografía. Disponible en: https://www.inegi.org.mx/programas/enigh/nc/2016/ (Consultado: 8/2/2019)

INEGI (2018). Actualización de la Medición de la Economía Informal, 2003-2017 preliminar, año base 2013. Comunicado de Prensa Núm. 658/18. Instituto Nacional de Estadística y Geografía. Disponible en: https:// www.inegi.org.mx/contenidos/saladeprensa/boletines/2018/StmaCntaNal/MEI2017.pdf (Consultado: $12 / 8 / 2019)$

INEGI (2019). Encuesta nacional sobre productividad y competitividad de las micro, pequeñas y medianas Empresas (ENAPROCE). Instituto Nacional de Estadística y Geografía. Aguascalientes, México.

Jang, S. \& Park, K. (2011). Inter-relationship between firm growth and profitability. International Journal of Hospitality Management, 30 (4), 1027-1035. https://doi.org/10.1016/j.ijhm.2011.03.009

Karlan, D. \& Zinman, J. (2011). Microcredit in theory and practice: Using randomized credit scoring for impact evaluation. Science, 332 (6035), 1278-1284. https://doi.org/10.1126/science.1200138

Korunka, C., Kessler, A., Frank, H. \& Lueger, M. (2011). Conditions for growth in one-person startups: A longitudinal study spanning eight years. Psicothema, 23 (3), 446-452.

Kuratko, D., Hornsby, J. \& Naffziger, D. (1999). The adverse impact of public policy on microenterprises: An exploratory study of owners' perceptions. Journal of Developmental Entrepreneurship, 4 (1), 81-92.

Markman, G., Baron, R. \& Balkin, D. (2005). Are perseverance and self-efficacy costless? Assessing entrepreneurs' regretful thinking. Journal of Organizational Behavior, 26 (1), 1-19. https://doi.org/10.1002/job.305

Monge, R. \& Torres, F. (2015). The dynamics of entrepreneurship in Costa Rica. An analysis of firm entry, exit, and growth rates. Discussion Paper, IDB-DP-367. Inter-American Development Bank. Disponible en: https:// publications.iadb.org/handle/11319/6784 (Consultado: 20/2/2018)

Mungaray, A., Ramírez, N., Aguilar, J. \& Beltrán, J. (2007). Poder de mercado en microempresas de Baja California. Problemas del Desarrollo, 38 (148), 173-194. https://doi.org/10.22201/iiec.20078951e.2007.148.7653

Nichter, S. \& Goldmark, L. (2005). Understanding micro and small enterprise growth, MicroREPORT 36, Accelerated Microenterprise Advancement Project: USAID. Disponible en: https://pdfs.semanticscholar. org/3993/ddc2dd810f88ab99a21f9d19321951435642.pdf (Consultado: 18/1/2019)

Rivera, R. (2018). Heterogeneidad en las microempresas informales mexicanas: evidencia empírica y algunas implicaciones de política. Perfiles Latinoamericanos, 26 (51), 63-87. https://doi.org/10.18504/pl2651-003-2018

Schindler, F. (2016). Is small better? IEEE Microwave Magazine, 17 (1), 16-18.

Simpson, B. (2001). Innovation and the micro-enterprise. International Journal of Services Technology and Management, 2 (3), 377-387. https://doi.org/10.1504/IJSTM.2001.001610

Texis, M., Ramírez, M. \& Aguilar, José G. (2016). Microempresas de base social y sus posibilidades de supervivencia. Contaduría y Administración, 61 (3), 551-567. https://doi.org/10.1016/j.cya.2015.04.001

Thapa, A. (2015). Determinants of microenterprise performance in Nepal. Small Business Economics, 45 (3), 581-594. https://doi.org/10.1007/s11187-015-9654-0

Webb, J., Tihanyi, L., Ireland, R. \& Sirmon, D. (2009). You say illegal, I say legitimate: Entrepreneurship in the informal economy. Academy of Management Review, 34 (3), 492-510. https://doi.org/10.5465/amr.2009.40632826 